\title{
Income Inequality in Pakistan: An Analysis of Existing Evidence
}

\section{ZAFAR MAHMOOD*}

To study the consequences of an economic change on income distribution we rank distributions of income at different points in time and quantify the degree of income inequalities. Changes in income distribution can be ascertained either through drawing the Lorenz curves or through estimating different inequality indices, such as Gini Coefficient, coefficient of variation, standard deviation of logs of incomes, Theil's Index and Atkinson's Index.

Ranking the distributions of income through Lorenz curves is, of course, possible only as long as they do not intersect. Moreover, when Lorenz curves do not intersect each other, all inequality measures rank income distributions uniformly. However, if the Lorenz curves do intersect each other, different inequality measures may rank income distributions differently ${ }^{1}$ and thus the direction of change cannot be determined unambiguously. For this reason, the use of a single measure would be misleading. Accordingly, the use of a 'package' of inequality measures becomes essential.

Most of the previous studies on income distribution in Pakistan measure income inequality by using Gini Coefficient, Theil's Index and Coefficient of Variation. ${ }^{2}$ These studies provide no explicit reason for preferring one measure to another. As a result, these studies do not give due consideration to the conceptual underpinnings of these measures, which are essential for understanding the implications of the results regarding various measures of income inequality.

Our main concern in this study is to compare the results regarding changes in income distribution derived from the various measures of inequalities. Such an analysis can be extended to analyse the impact of any income/asset redistribution policy.

The schematic details of the study are as follows. Section I deals with methodological and data issues. Section II contains empirical results of the study. Finally, Section III presents conclusions and recommendations.

*Research Economist, Pakistan Institute of Development Economics, Islamabad (Pakistan).

${ }^{1}$ Different inequality measures give different weights to income transfers at different income levels.

${ }^{2}$ These studies are: Alauddin [1], Ayub [3], Azfar [4], Bergan [5], Chaudhry [6], Jeetun [9] and Khandkar [11]. For a detailed review of these studies, see Kemal [10]. 


\section{METHODOLOGICAL ISSUES}

Dalton $[2 ; 7]$ has laid down the following two principles for a satisfactory measurement of inequality:

(i) Inequality index should remain unchanged relative to proportional increases in all income (i.e. index is independent of mean). It is known as Dalton's Principle of Population.

(ii) A strictly positive transfer from a rich person to a poor person ought to lead to a reduction in inequality index. ${ }^{3}$ It is known as Dalton's Principle of Transfers.

We shall analyse various measures of income inequality in terms of the above two principles and will discuss the various properties of these measures.

To depict income distribution, Lorenz [17] devised a diagram, in which percentages of population from the lowest income group to the highest income group are represented on the horizontal axis and the percentage of income received by the bottom ' $\mathrm{X}$ ' percent of the population is shown on the vertical axis. This curve gives the relation between the cumulative percentage of the income recipients and the cumulative percentage of income. If the curve is equidistant from the two axes, it coincides with the line of equality - a situation of absolute equality of all incomes. If only one person gets the whole income, then the curve will coincide with the bottom axis and the right hand vertical axis. Lorenz curve traces the pattern of income distribution at a given point of time. As long as the Lorenz curves corre sponding to different points of time do not intersect, we can unambiguously determine the direction of change in income distribution. However, when the two Lorenz curves intersect, it is difficult to say whether the income distribution has improved or worsened.

Gini's concentration ratio $(G)$ can best be explained through Lorenz curves and can be computed by the following formula [8] :

$$
\begin{gathered}
G=1-\sum_{i=1}^{n} S_{p i}\left(y_{i}+y_{i-1}\right) \\
0 \leqslant G \leqslant 1
\end{gathered}
$$

where $S_{p i}$ is the population share of the $i$ th income group and $y_{i}$ is cumulated in come share of the $i$ th income group.

Since population is concentrated around the mean income, Gini attaches more weight to transfers affecting middle income classes and not much weight to changes in the extreme income classes. Gini coefficient ignores intragroup inequalities in incomes, and as such it understates income inequalities.

${ }^{3}$ This property rejects the measures such as relative mean deviation and the interquartile range, because they are unaffected by transfers on the same side of the mean.
Variance attaches the highest weight to transfers taking place at the extreme income ranges. However, since the variance is not independent of unit of measurement, a proportional increase in income across the board will be shown by variance as an increase in income inequality while it has remained constant. Therefore, it fails to satisfy the Principle of Population. A better measure which does not suffer from the deficiency is the coefficient of variation $(C . V$.$) , which is defined as$

$$
C . V .=\frac{\text { Standard Deviation of Incomes }}{\text { Mean Income }}
$$

The coefficient of variation is sensitive to income transfers for all income levels and is independent of the mean income level. The coefficient of variation attaches equal weights to transfers of income at different income levels, i.e. the impact of redistribution from one income group to another income group would be the same, irrespective of the level of income. It is, however, possible that the impact of a transfer would be greater at a lower income level than at the high income level.

If one wishes to attach greater importance to income transfers at lower end, then the neutrality property of coefficient of variation poses serious doubts. A measure of inequality that gives more importance to lower income levels is the standard deviation of logs of incomes (S.D.):

$$
S . D .=\left[\sum_{i=1}^{n}\left(\overline{\log Y}-\log Y_{i}\right)^{2} 1 / n\right]^{1 / 2}
$$

where $Y_{i}$ is average income of the $i$ th income group, $Y$ is economy-wide mean income and $n$ is the number of income groups.

A logarithmic transformation reduces the deviation and highlights the differences at the lower end of the income scale. Therefore, this measure has relevance if inequalities at the lower income levels are important. However, as income levels increase, the logarithmic values shrink so rapidly that we may find S.D. even rising when there are income transfers from the rich to the poor and thus failing to satisfy the Principle of Transfers.

Theil [18] introduced an inequality measure which is derived from the notion of 'entropy' in information theory. The basic idea behind the 'entropy' is that occurrences which differ greatly from what was expected should receive more weight than events which conform with prior expectations. Thus entropy index is the expected information content of each outcome. Theil's index can be presented as follows:

$$
T=\log N-\sum_{i=1}^{n} Y_{i} \log 1 / Y_{i}
$$

where $Y_{i}$ is income share of the $i$ th income group and $N$ is the number of income 
groups. When $T=0$, we get complete equality and when $T=\log N$, we get complete inequality. This index can be useful if inequality in the high or medium ranges is more important.

As mentioned earlier, different inequality measures attach different weights to transfers taking place at different income levels. These weights are the implicit values that each measure embodies for a desirable distribution of income. Atkinson [2] argues that the conventional measures should be rejected in favour of direct consideration of the values we should like to see embodied. Atkinson defines the "equally distributed equivalent level of income" as "the level of income per head which, if equally distributed, would give the same level of social welfare as the present distribution" [2, p. 250]. Atkinson's Index can be put as:

$$
\begin{aligned}
A_{t}= & 1-\left[\sum_{i=1}^{n}\left(\bar{Y}_{i} / \bar{Y}\right)^{1-\epsilon} f\left(Y_{i}\right)\right]^{1 / 1-\epsilon} \text { for } \epsilon \$ 1 \\
& 0 \leqslant A_{t} \leqslant 1
\end{aligned}
$$

where $Y_{i}$ is mean income of the $i$ th income class; $Y$ is economy-wide mean income; $f\left(Y_{i}\right)$ is percentage of population in the $i$ th income class; and $\epsilon$ is inequality aversion parameter.

The inequality aversion parameter, $\epsilon$, represents the weight attached by society to inequality in the distribution: high values of $\epsilon$ mean that the society is particularly averse to inequality, ${ }^{4}$ whereas a zero value means that it is indifferent to inequality.

\section{Data Issues}

We have made use of the surveys conducted and compiled by the Federal Bureau of Statistics. Although commendable efforts have been made to ensure the representativeness of the data and to minimize the sampling errors, yet the data suffer from certain drawbacks which should be kept in mind when drawing any implications from the results of the present study.

A major shortcoming of the survey data is the small sample size for the highest income group which leads to an understatement of the incomes of the group. This problem arises owing to non-response and understatement of incomes. Moreover, the data do not include the corporate retained earnings, which leads to a further understatement of income.

To pool rural-urban data, weights of 1962 are used, which do not consider the effect of increased urbanization and emergence of new urban areas and hence

${ }^{4}$ It should be mentioned here that Atkinson's measure violates the basic assumption of strict concavity on either extremum, where welfare derived from a change in income remains same. would give lower weights to urban inequalities. The 1979 survey data are simply pooled without assigning any weights to rural and urban areas. Therefore, by according proper weights to rural and urban areas, we have pooled the data for total Pakistan.

We made an attempt to remove the understatement of incomes in the data for the highest income group by splicing the income tax data in the urban areas. Even the use of the income tax data did not affect the results, ${ }^{5}$ because of the narrow coverage of the income tax data [12] and the widespread evasion of income tax. Moreover, the data given in income tax statistics are for the assessable income. Because of the various allowances which are not uniformally available to the taxpayers, it is difficult to determine the income of each taxpayer from his assessed income.

Income groups given in the Household Income and Expenditure Surveys are fewer for 1963-64 and 1979 [13;16] than for the rest of the period. Moreover, class intervals of different income groups for each year are not equal. Such inconsistencies may create some problems, especially when the comparison of income distribution is made over time. To overcome any such problem, we have transformed the whole data into income deciles by the linear-interpolation method.

\section{EMPIRICAL RESULTS}

Analysis of Income Shares

We start analysing results by ordinal ranking of the household income distribution in rural, urban and total Pakistan.

Table 1 presents the income share of each household decile for the rural areas of Pakistan. The table reveals that income share of up to the 7 th decile increased between 1963-64 and 1968-69, while those of the 8th, 9th and 10th deciles decreased over the same period. However, in the intervening period there was some fluctuation in the income shares of various deciles. For example, in 1968-69, the income shares of the 8th and 9th deciles increased over those for 1966-67. A comparison of income distribution in 1969-70 with that in 1968-69 reveals an increase in the income share of the 10th decile, but a decrease or stagnation in the income shares of all the other deciles. However, if we compare $1969-70$ with the earlier period, i.e. 1963-64 and 1966-67, we see an increase in the income shares of all the deciles except the 10th decile. If we compare 1970-71 with any other period under consideration, we see that this was the period when income was more equally distributed; all the poor and middle-income classes observed an increase in their income

${ }^{5}$ Owing to insignificant improvement in our results, we did not report these results in
this study. 
+ $\infty$ m ha

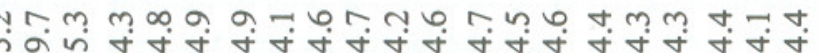

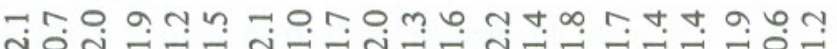

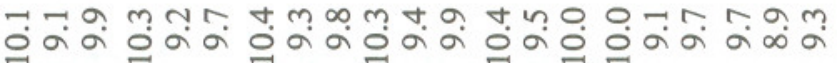

shares, and the rich classes observed a decrease in their income share. The picture in 1971-72 was completely different from that in 1970-71 when the only increase in income share was experienced by the households of the 10th decile: all other deciles recorded a decrease in their income share. Also, compared to 1971-72, we observed a further decrease in income share of up to the 7th decile in 1979, while the 8th and 10 th deciles recorded an increase in their income shares.

It can be seen from Table 1 that in urban Pakistan income shares of the bottom 80 percent of households have increased over the period from 1963-64 to 1969-70. The income share of the 9th decile decreased up to 1968-69, but afterwards it showed a rising trend. The share of incomes of the richest 10 percent of the households increased up to $1968-69$, but it went down in the following year. Income shares of both the poorest and the richest 10 percent of the households declined in 1970-71, while those of the remaining households showed either a slight increase or stagnation. In contrast with $1970-71$, both the poorest and the richest 10 percent households in 1971-72 recorded an increase in their income shares while income shares of the rest of the households went down. The year 1979 saw the most un equal income distribution of the whole period under consideration when only the richest 10 percent of the households showed an increase in their income share and all other households experienced a considerable fall in their income shares.

It can be seen from Table 1 for total Pakistan that during the period from 1963-64 to 1970-71, the bottom 70 percent of the households saw an increase in their income shares. The 8th and the 10th deciles had a mixed trend while the 9th decile witnessed a decrease in their income shares. As compared to 1970-71, only the richest 10 percent households in 1971-72 recorded an increase in their income share while all other households experienced a decrease. However, income distribution in 1971-72 appeared to be more in favour of both the poorest and the richest households as was observed in 1963-64 and 1966-67. As compared to 1971-72, the lowest 70 percent households in 1979 registered a further decrease in their income shares while the rest of the households had an increase in their respective shares.

It is difficult to find any ranking of the distributions by analysing income shares. Because different inequality measures give different weights to transfers to certain income levels, there is a possibility that different inequality measures may lead to different ranking of the income distributions.

\section{Ranking of Income Distributions}

Although the analysis of income shares did not clearly show which year's distribution was more equal, estimates of most of the inequality indices reported in Table 2 for rural Pakistan do show a uniform ranking of income distributions over 
the entire period under consideration. ${ }^{6}$ It can be seen from Table 2 that inequalities went down over the 1963-64 - 1968-69 period because the poor and the middle income classes enjoyed an increase in their income shares during this period. In the following year (1969-70), a slight increase in inequality was observed, which was reversed in 1970-71, when all the poor and the middle-income classes enjoyed an increase in their income shares. However, this trend could not last long in 1971-72, when inequality went up owing to the only increase in income share of the richest 10 percent households. A rising trend in inequality was also observed in 1979 when the poor and the middle-income classes suffered a further decrease in their income shares.

Estimates of inequality indices for urban areas reported in Table 2 show divergent trends in income distributions. For example, on the basis of Gini Coefficient, Atkinson's Index for $\epsilon=0.5$ and Theil's Index, the inequality was at the lowest level in 1969-70. However, on the basis of the standard deviation of the logs of incomes and Atkinson's Index for $\epsilon=3.0$, it was the year 1971-72 in which the inequalities in income distribution were the least, while the coefficient of variation suggests that the inequality was at the minimum in 1970-71. Therefore, without any reference to the welfare functions, it is difficult to conclude whether the degree of inequality has improved or worsened.

It is interesting to note that while all other indices for urban areas showed an increase in inequality in 1971-72 over that in 1970-71, Atkinson's Index for $\epsilon=3.0$ and the standard deviation of the logs of incomes showed a decrease in inequality This is due to the fact that only the poorest and the richest households enjoyed an increase in their income share but the change in the income share of the poorest households was more than that of the richest households. The Atkinson's Index for $\epsilon=3.0$ and the standard deviation of the logs of incomes share the property of assign ing more weights to transfers to the lower income groups. That is why both the measures highlighted this change as a decrease in inequalities although the income share of the richest class had also increased.

For urban areas, both the Theil's Index and Atkinson's Index for $\epsilon=0.5$ move in the same direction in five out of seven years, and the Gini Coefficient and the coefficient of variation show very divergent trends. It is worth noting that while for the year in which income inequality was the least there was no agreement among in equality measures, they all agree for the period in which inequality was the maximum, viz. 1979. This probably is due to the fact that the richest 10 percent of the households received most of the income.

${ }^{6}$ It should be mentioned here that the standard deviation of logs of income and Atkinson' Index for $\epsilon=3.0$ disagree with other indices in the cases of a few years. 
In the case of total Pakistan, all the inequality measures agree in ranking 1970-71 as the year of greater equality and 1963-64 as the year of greater inequality. ${ }^{7}$ However, we do observe some disagreement on ranking for the rest of the period. It can be seen from Table 2 that Gini Coefficient, Coefficient of Variation and Atkinson's Index for $\epsilon=0.5$ provide the same ranking except that the Coefficient of Variation strikes a discordant note for 1966-67. Similarly, Atkinson's Index for $\epsilon=3.0$, the standard deviation of the logs of incomes and Theil's Index provide the same ranking.

\section{CONCLUSIONS AND RECOMMENDATIONS}

Besides discussing the properties of measures of income inequality, we have presented, in this study, the estimates of these measures to help analyse changes in the income distribution. The results show that reliance on the use of a single measure may lead us to erroneous conclusions. While in the case of the rural areas of Pakistan, most of the inequality measures will give us the correct direction underlying the changes in income distribution, any reliance on Gini coefficient or any other single measure of income inequality in the absence of a certain welfare function will yield misleading results for the urban areas and hence for total Pakistan.

The analysis shows a declining trend in income inequalities for both the rural and urban Pakistan up to the year 1970-71 but a rising trend soon afterwards. While in the rural areas the income inequality in 1979 was still lower than that in 1963-64, the income inequality in the urban areas in 1979 was at the maximum level.

It should be noted that income inequalities in urban areas have always been much higher than those observed in the rural areas. As a matter of fact, the difference in inequalities in the two areas has even widened. This is disturbing, because the inequalities have grown faster in those areas which are fast expanding, with the result that income inequalities have worsened over time.

From the trends of the inequalities observed in Pakistan one can question the distribution of benefits of growth which the country has achieved over time. From these trends, it seems that Kuznet's thesis is very much valid for the urban areas of Pakistan if not for the rural areas. All the industrial growth taking place in urban areas has gone into the hands of the urban elite. This situation is a cause for serious concerns as the urban sector of Pakistan is growing rapidly, and if urban inequalities also grow with it, then there are chances of a further deterioration of the situation. ${ }^{8}$

${ }^{7}$ The standard deviation of the logs of income and Atkinson's Index $\epsilon=3.0$ are exceptions, which report 1963-64 as the period of high inequality.

${ }^{8}$ We may also note that these results emerge despite the fact that the survey data leave out the big capitalists who have been the main beneficiaries of the industrial growth during the Sixties and also later.
These trends in inequality give an indication of a worsening situation to the policy makers and require that redistributive policies be formulated in favour of the poor and middle-income classes.

We may note that the feeling that inequalities in income in Pakistan have been high is suggested by the fact that wages have increased less rapidly than the per capita income. However, one should remember that such an analysis is related to the functional distribution of income and not to the size distribution of income. The two can be related only if we have data on assets, which unfortunately do not exist in Pakistan. A study of size distribution of income, coupled with asset distribution, can prove more fruitful.

\section{REFERENCES}

1. Alauddin, T. "Mass Poverty in Pakistan: A Further Study". Pakistan Development Review. Vol. XIV, No. 4. Winter 1975.

2. Atkinson, A. B. "On the Measurement of Inequality". Journal of Economic Theory. Vol. 2. 1970.

3. Ayub, M. A. "Income Inequality in a Growth - Theoretic Context: The Case of Pakistan". Ph.D. Thesis submitted to Yale University. 1977.

4. Azfar, J. "The Distribution of Income in Pakistan: 1966-67". Pakistan Economic and Social Review. Vol. XIV, Nos. 1-4. 1976.

5. Bergan, A. "Personal Income Distribution and Personal Savings in Pakistan". Pakistan Development Review. Vol. VII, No. 2. Summer 1967.

6. Chaudhry, M. Ghaffar. "Green Revolution and Redistribution of Rural Incomes: Pakistan's Experience". Pakistan Development Review. Vol. XXI, No. 3. Autumn 1982.

7. Donaldson, D., and John A. Weymark. "A Single-Parameter Generalization of the Gini Indices of Inequality”. Journal of Economic Theory. Vol. 22, No.1. February 1980.

8. Jain, S., and A. E. Tiemann. Size Distribution of Income: Compilation of Data. Washington, D.C.: I.B.R.D., Development Research Center. August 1973. (Discussion Paper No. 4)

9. Jeetun, A. "Trends in Inequality of Income Distribution in Pakistan". Karachi: University of Karachi, Applied Economic Research Centre. September 1978. (Discussion Paper No. 29)

10 Kemal, A. R. "Income Distribution in Pakistan: A Review". Islamabad: Pakistan Institute of Development Economics. April 1981. (Research Report Series No. 123)

11. Khandkar, R. H. "Distribution of Income and Wealth in Pakistan". Pakistan Economic and Social Review. Vol. XIV, Nos. 1-4. 1976. 
12. Pakistan. Central Board of Revenues. All Pakistan Income Tax Reports and Returns. Islamabad. (Various years)

13. Pakistan. Economic Affairs Division. Central Statistics Office. Report on the Quarterly Survey of Current Economic Conditions in Pakistan: Household Income and Expenditure (July 1963 to June 1964). Karachi. 1967.

14. Pakistan. Economic Coordination and External Assistance Division. Central Statistical Office. Report on the Quarterly Survey of Current Economic Conditions in Pakistan: Household Income and Expenditure Survey (July 1966 to June 1967). Karachi. 1971.

15. Pakistan. Ministry of Finance, Planning and Development. Statistical Division. Household Income and Expenditure Survey, 1968-69, 1969-70, 1970-71 and 1971-72. Karachi. 1973.

16. Pakistan. Statistics Division. Federal Bureau of Statistics. Household Income and Expenditure Survey, 1979. Karachi. 1983.

17. Sen, A. On Economic Inequality. Oxford: Clarendon Press. 1973.

18. Theil, H. Economics and Information Theory. Amsterdam: North-Holland. 1967. 


\section{Comments on \\ "Income Inequality in Pakistan: An Analysis of Existing Evidence"}

This is purely a statistical paper. The author studies the pattern of income inequality in Pakistan, using several inequality measures. The reader's expectations are aroused with the promises of being provided with an 'explicit reason for preferring one measure rather than another' as well as potential in the analysis for extension to study the impact of any income or asset redistribution policy. But what is provided by way of 'explicit reason' is simply a catalogue of formulas and their properties. This purpose could well have been served if the author had simply guided the reader to standrad works on the subject, such as those by Sen [1] and Szal and Robinson [2] The other claim, too, is an overstatement. Extensions of the formulas reported in this study whereby one can readily gauge the impact of redistribution policies still elude economists. These, however, are not the main reasons for this study on the author's part.

The author bases his theoretical argument for taking into consideration more than one summary measure of income inequality on the possibility of the Lorenz Curves for various years intersecting one another. An appropriate statistical response in such a case would have been the estimation of the functional forms of income distributions with the help of such relative-frequency functions as lognormal, gamma or beta densities. This is so because an increase in the number of the parameters at one's disposal to describe income distributions in various years is most likely to enhance one's ability to identify the direction of change in income inequality. No doubt, Atkinson does point to a way out of the dilemma situations by means of the Atkinson's Index. However, such an index depends on the existence of a particular welfare function which, too, does not carry to its credit the consensus supposedly absent in the case of inequality measures, such as the Gini coefficient, when Lorenz Curves for different years do intersect. The functional-form approach has considerable promise in this regard.

The author's case for relying on a basket of summary measures is weak on empirical grounds too. One can form a priori judgements about the course of overall income inequality from one data year to the next on the basis of the decile estimates 
reported by the author in Table 1 of the paper. For example, in the rural areas there seems to have been a redistribution away from the three top deciles to all the seven lower deciles in 1966-67, as compared with 1963-64. ${ }^{1}$ Thus the year 1966-67 is a clear-cut case of decline in income inequality. Similarly, one can make the following observation for 1969-70, as compared with 1968-69. Below the median, there is no change in income shares of the first, fourth and fifth deciles, and there are compensating changes between the second and third deciles. But, above the median, there is a redistribution away from the sixth, seventh, eighth and ninth deciles toward the tenth decile. In this situation, one would expect overall income inequality to have gone up in 1969-70 as compared with 1968-69. This reasoning can be extended to all the data years, for both rural and urban areas. As a result, one may envisage the following pattern of income inequality in rural and urban areas:

Change in Income Inequality as compared with the Previous Data Year

\begin{tabular}{lcccccc}
\hline & $1966-67$ & $1968-69$ & $1969-70$ & $1970-71$ & $1971-72$ & 1979 \\
\hline Rural & $\downarrow$ & $\downarrow$ & $\uparrow$ & $\downarrow$ & $\uparrow$ & $\uparrow$ \\
\cline { 2 - 7 } Urban & $\downarrow$ & $\downarrow$ & $\downarrow$ & $\uparrow$ & $\uparrow$ & $\uparrow$ \\
\hline
\end{tabular}

Note: $\downarrow$ implies decrease and $\uparrow$ increase.

If one compares the coefficient estimates reported in Table 2 of the paper for the rural category, all the measures rank all income distributions for rural areas in line with this stipulated pattern. In the case of the urban areas, none of the Standard Deviation of log income, Coefficient of Variation, Atkinson's Index (for both $\epsilon=0.5$ and $\epsilon=0.3$ ) and Theil's Index provides a ranking in conformity with the expected ranking. Surprisingly, only the Gini Coefficient correctly gauges the expected pattern of income inequality for urban areas also. This is certainly not what the reader is led to believe in this paper. Leaving the justification issue aside, let us turn to a more fundamental problem in the paper. This concerns the interpolation procedure adopted by the author to derive the decile estimates on which the income inequality parameter estimates, in turn, are based.

According to an earlier version of this paper, the author adopts the route of mean income in order to arrive at the percentage of total income accruing to various deciles of households. This is done by means of a two-stage interpolation procedure.

${ }^{1}$ In principle, the term "redistribution" is to be used with reference to a given mean income, but in a time-series framework it is used with reference to aggregate and, hence, mean income change. Thus the use of the term here is ad hoc. It is just to facilitate comparative statements about the degree of income inequality between two years.
This method utilizes the following information: the lower income bound $f\left(x_{1}\right)$ of the income group containing a decile point, its upper income bound $f\left(x_{2}\right)$ and its mean income $f\left(x_{\mathrm{o}}\right)$, where $x$ is a cumulative percentage of households and $f\left(x_{\mathrm{o}}\right)$ lies between $f\left(x_{1}\right)$ and $f\left(x_{2}\right)$. The problem at hand is to find mean income $f\left(x^{*}\right)$ for the $x^{*}$ decile, where $x^{*}$ lies between $x_{1}$ and $x_{2}$. In the first stage, points $\left(x_{1}, f\left(x_{1}\right)\right)$ and $\left(x_{2}, f\left(x_{2}\right)\right)$ are used to determine $x_{0}$, the cumulative percentage of households corresponding to the known mean income $f\left(x_{0}\right)$. In the second stage, either points $\left(x_{1}, f\left(x_{1}\right)\right)$ and $\left(x_{0}, f\left(x_{0}\right)\right)$ or $\left(x_{0}, f\left(x_{0}\right)\right)$ and $\left(x_{2}, f\left(x_{2}\right)\right)$, depending on whether $x^{*}$ falls between $x_{1}$ and $x_{0}$ or between $x_{0}$ and $x_{2}$, are used to find the desired $f\left(x^{*}\right){ }^{2}$ In each of the two stages, a straight-line interpolation method is used. There is a basic problem with this method in its application to the income distribution data. It does not enable one to carry out mean-preserving adjustments in the data. In other words, no decile pattern constructed with the help of this method can be claimed to belong to the original income distribution which it is supposed to represent. In the presence of this anomaly, the income inequality estimates reported in Table 2 are meaningless. Thus there is a need to re-do the entire exercise with the help of the standard linear-interpolation procedure, using data on cumulative percentage of income received along with cumulative percentage of households - without any reference to the mean income data.

Associate Professor,

International Institute of Islamic Economics,

Islamic University,

Islamabad

\section{REFERENCES}

1. Sen, A. K. On Economic Inequality. Oxford: Clarendon Press. 1973.

2. Szal, R., and S. Robinson. "Measuring Income Inequality". In C. R. Frank, $\mathrm{Jr}$., and R. C. Webb (eds.), Income Distribution and Growth in the LessDeveloped Countries. Washington, D.C.: The Brookings Institution. 1977.

${ }^{2} \mathrm{My}$ interpretation of the second stage is based on my discussions with the author on this the conference, formula reported problem noted here remains. It may also be mentioned that according to the formula reported by the author, the likelihood of negative weights is very much there, whenever $x_{1}$ is greater than $x_{0}$. Such weights are hard to rationalize, to say the least. 